\title{
Two commentaries on interventions for the management of temporomandibular joint osteoarthritis
}

\author{
Abstracted from \\ de Souza RF, Lovato da Silva CH, Nasser M, Fedorowicz Z, Al-Muharraqi MA. \\ Interventions for the management of temporomandibular joint osteoarthritis. \\ Cochrane Database Syst Rev 2012; 4: Art. No. CD007261. \\ Address for correspondence: Luisa Fernandez Mauleffinch, Review Group Co-ordinator, \\ Cochrane Oral Health Group, MANDEC, School of Dentistry, University of Manchester, \\ Higher Cambridge Street, Manchester, M15 6FH, UK. E-mail: luisa.fernandez@manchester.ac.uk
}

\author{
Question: In patients with temporomandibular joint osteoarthritis (TMJ OA) what is the best \\ surgical or non-surgical treatment?
}

Data sources The Cochrane Oral Health Group Trials Register, Cochrane Central Register of Controlled Trials (CENTRAL), Medline, Embase and PEDro databases were searched with no language restrictions. Handsearching of a number of relevant dental journals and the reference lists of identified articles was also conducted.

Study selection Randomised controlled trials (RCTs) comparing any form of non-surgical or surgical therapy for TMJ OA in adults were included. Only studies with clinical and/or radiological diagnosis of TMJ OA according to the Research Diagnostic Criteria for Temporomandibular Disorders (RDC/TMD) guideline or compatible criteria were considered. Pain/tenderness/discomfort in the TMJs or jaw muscles, self assessed range of mandibular movement and TMJ sounds were the primary outcomes considered. Secondary outcomes included the measurement of quality of life or patient satisfaction, morphological changes of the TMJ and TMJ sounds

Data extraction and synthesis Study selection, data abstraction and risk of bias assessment were carried out independently by two reviewers. Meta-analysis was not conducted owing to clinical heterogeneity.
Results Although three RCTs were included in this review, pooling of data in a meta-analysis was not possible due to wide clinical diversity between the studies. The reports indicate a not dissimilar degree of effectiveness with intra-articular injections consisting of either sodium hyaluronate or corticosteroid preparations, and an equivalent pain reduction with diclofenac sodium as compared with occlusal splints. Glucosamine appeared to be just as effective as ibuprofen for the management of TMJ OA.

Conclusions In view of the paucity of high level evidence for the effectiveness of interventions for the management of TMJ OA, small parallel group RCTs which include participants with a clear diagnosis of TMJ OA should be encouraged and especially studies evaluating some of the possible surgical interventions.

This paper is based on a Cochrane Review published in the Cochrane Library 2012, issue 4 (see www.thecochranelibrary.com for information). Cochrane Reviews are regularly updated as new evidence emerges and in response to feedback, and the Cochrane Library should be consulted for the most recent version of the review.

Limited evidence for the effectiveness of interventions for the management of temporomandibular joint osteoarthritis

Osteoarthritis of the Temporomandibular Joint (TMJ OA) is a debilitating degenerative chronic disease than can limit patients' masticatory ability and impact quality of life when pain is associated. It is characterised by morphological changes within the articular surface and loss of protective lubrication from the synovial fluid that may lead to degeneration with noises and sounds in the TMJ during function. TMJ OA tends to present in the elderly or patients with history of trauma and functional overload or dysfunctional remodeling. It may be associated with intra-articular derangements or myofascial dysfunction and pain. Therapies for TMJ OA are vast and varied depending on the severity and subjective to patients' reported symptoms. Treatment protocols target pain and inflammation reduction, rehabilitation of joint function/mobility, prevention of further damage and disability.

The authors of this paper attempted to study the effectiveness of some therapies. Non-surgical management includes but is not limited to oral appliances, pharmacological management of pain and inflammation with anti-inflammatory agents and muscle relax- ants, physiotherapy, massage therapy, ultrasound, electrogalvanic stimulation, lifestyle modification with control of parafunction and overload, diet modification and supplementation and relaxation. Surgical management depending on OA severity involves intraarticular injection with anti-inflammatory agents, arthrocentesis, arthroscopy, arthroplasty and joint replacements.

The authors of this well formatted systematic review attempted to demonstrate the effectiveness of different therapies for the treatment of TMJ OA. The review has a defined purpose, inclusion and exclusion criteria and types of outcome measures. However, they didn't clearly define limits for short-term or long-term outcome measures for specified therapies. They classified the participants to be adults, legally defined as being greater than 18 years of age.

Prevalence of $\mathrm{OA}$ in 18-year-olds can be questionable as they may still be in a condylar growth phase. OA increases with age. Length of time for the interventions is important in prevention of disease progression for a younger vs elderly patient. Gender/hormonal effects 
were not looked at because of extent of heterogeneity amongst the few qualifying studies.

The authors found that there were a large number of studies that included mixed groups with participants diagnosed with TMJ OA and other TMJ disorders. It should be noted that perhaps the OA doesn't occur alone and is a co-morbid disease process associated with other joint dysfunctions or conditions. OA could therefore be the end result of intra-capsular derangements and thus must be looked at concomitantly with other associated conditions. This may be a reason for the limited availability of the data.

The authors sorted through databases where they would find the best quality of studies and RCTs and excluded studies that were not RCTs. They hand selected specific journals to include in their search that were not found in the databases searched. They attempted to search for unpublished data by contacting authors of conducted studies for further information. They also included all languages and found translators to ensure that all available papers were included in this study. Two independent reviewers assessed the quality of the research by graded criteria and discussed any disagreements with a third party consultant.

The authors were limited by the available randomised clinical trials (RCTs) and only three studies qualified for this review with diverse interventions making it difficult to assess any long-term recommendation in therapeutic outcomes. Use of standardisation of diagnostic criteria, as RDC/TMD ${ }^{1-3}$ are needed for research comparisons between studies. Many studies were excluded because diagnosis wasn't based on this standard. There was found to be limited availability of the trials with all therapeutic modalities. It is unclear how they reached a total of three studies or four full-text articles with one study awaiting assessment. There was no further mention of the one study awaiting assessment.

Of the three included studies two did not contain control groups to evaluate the benefits of therapy over no treatment at all. All three considered pain/tenderness of TMJ and muscles of mastication or mandibular movement as outcomes. Not all measured joint sounds or radiographic changes on CT scans. Not all parameters of outcomes were tested in the three studies, limiting the availability of data for this review and making compari- son between nonsurgical and minimally invasive interventions inapplicable. Therapeutic targets (long term outcomes) were unspecified and sequential therapy was not given any mention. Only between 29-45 participants were included in these trials. Appropriately, all participants underwent CT scans for assessments. Weakness with biases within the studies varied with one demonstrating reporting bias and others with uncertain biases in selection, performance and detection.

They were unable to combine the studies yet they conducted statistical analysis of the individual studies that were not statistically significant to prove any one therapeutic advantage over another.

The surgical outcomes were not available for assessment and thus no conclusion can be made of their therapeutic benefit. There is insufficient evidence to conclude any one therapeutic advantage over another. From the results of this review all therapies were equally effective in pain reduction. In totality there is lack of evidence for assessment of all outcomes listed in this review by the authors. In two of the reviewed studies only pain and jaw movement were assessed and long-term outcomes were not available to assess factors such as prevention/progression of disease and quality of life improvements. Reports of side effects/intolerances of the diclofenac and occlusal splints could not be compared to the side effects/intolerances of other treatment modalities either because data were not available or because lack plausibility of comparison of effects unrelated to other therapies. This issue was not discussed in this review. Application of these limited therapeutic modalities to TMJ OA cannot be conclusively recommended, as harm was not assessed and benefits were short term.

\section{Dharti N. Patel}

\section{NYU, College of Dentistry, New York, USA}

1. Ahmad M, Hollender L, Anderson Q, et al. Research diagnostic criteria for temporomandibular disorders (RDC/TMD): development of image analysis criteria and examiner reliability for image analysis. Oral Surg Oral Med Oral Pathol Oral Radiol Endod 2009; 107: 844-860.

2. Dworkin SF. LeResche L. Research diagnostic criteria for temporomandibular disorders: review, criteria, examinations and specifications, critique. / Craniomandib Disord 1992; 6: 301-355.

3. Look JO, John MT, Tai F et al. The Research Diagnostic Criteria For Temporomandibular Disorders. II: reliability of axis I diagnoses and selected clinical measure. J Orofac Pain 2010; 24: 25-34.

\section{Few trials to support treatments for temporomandibular joint osteoarthritis}

The field of temporomandibular joint (TMJ) disorders has been receiving growing attention over the past few years, with several systematic reviews summarising many issues concerning their diagnosis and treatment. This review ${ }^{1}$ aimed to meta-analyse the data from randomised controlled trials (RCTs) on the management of TMJ osteoarthritis (OA), as diagnosed with the Research Diagnostic Criteria for Temporomandibular Disorders (RDC/TMD). ${ }^{2}$

The intention of the authors was very interesting and the resulting paper is a useful source of hints for readers willing to get deeper into the issue. The aim was clearly stated, the literature search comprehensive of five databases with no language restrictions plus hand-made search within some journals, and the data selection and extraction were performed meticulously. As recommended by the guidelines for reporting systematic reviews, a flow diagram of the articles identified, screened after removal of duplicate studies, assessed for eligibility, included in qualitative synthesis, and included in the review was provided. Only a few additional papers, mainly related with TMJ injections, were published after this review, but their findings are not suggestive of any relevant additional clinical information. ${ }^{3-5}$

Only three studies went on into the review process, and their wide clinical diversity (ie, the three studies compared 1.TMJ injections with hyaluronic acid vs. corticosteroids; or 2 . Diclofenac vs. occlusal splints; or 3. Glucosamine vs. ibuprofen) prevented the authors from performing meta-analysis of data. In summary, no scientifically sound conclusions based on multiple papers could be drawn, and small parallel group RCTs, possibly evaluating surgical interventions for TMJ OA, are recommended.

These conclusions are pertinent with the reviewed papers. Anyhow, it must be pointed out that, while focusing on strictly 
selective inclusion criteria to increase the internal validity of findings, the authors did not consider some disease-related methodological shortcomings leading to the exclusion of potentially clinically-relevant investigations, thus creating a super-selected pool of reviewed papers with low external validity. ${ }^{6}$ The literature on temporomandibular disorders (TMD) suggested that multiple diagnoses are frequent, ${ }^{7}$ and performing an 'ideal' study on 'pure' OA patients, as suggested by the authors, may be difficult and not logical from a clinical viewpoint. So, the exclusion criteria indi-

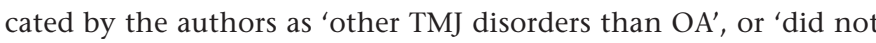
provide separate data for participants with $\mathrm{TMJ} \mathrm{OA}^{\prime}$, which were responsible for the wide majority of the excluded papers (31 out of 40 studies) need to be re-appraised with further assessment performed by reviewers with a more specific know-how of the TMJ disorders literature. Also, the assessment of the complex multimodal pain experience, taking into account the so-called RDC/TMD axis II at the time of group randomisation in RCTs, is a fundamental requisite for a real matching of any study groups. Moreover, it must be borne in mind that surgical interventions are hardly comparable with other treatment interventions in a true clinical trial for obvious methodological and ethical reasons.

All these observations suggest that, as a general remark, the conclusions of all literature reviews are strongly influenced by the inclusion criteria for study selection that, in turn, depend on the specific clinical expertise of the examiners managing the data. For this reason, it is not surprising that some lower-level, more narratively-oriented, systematic reviews are still widely appreciated by the researchers belonging to the specific field of interest. Having made these observations, there are no doubts that the authors should be complimented for their ambitious attempt to summarise such a vast issue, and this review may represent a stimulus for performing high-

\section{Practice point}

- TMJ osteoarthritis is common within temporomandibular disorder patient populations

- Several treatment approaches have been proposed over the years, with only a few high-quality RCTs comparing the different modalities

- It is reasonable that symptom management is considered the first-level therapeutic approach

- Further RCTs are needed, possibly taking into account for the multifaceted pain experience.

er-level investigations comparing the different strategies to manage TMJ osteoarthritis.

\section{Daniele Manfredini}

Department of Maxillofacial Surgery, University of Padova, Italy

1. de Souza RF, Lovato da Silva CH, Nasser M, Fedorowicz Z, Al-Muharraqi MA. Interventions for the management of temporomandibular joint osteoarthritis. Cochrane Database Syst Rev 2012; 4: Art. No. CD007261.

2. Dworkin SF, LeResche L. Research diagnostic criteria for temporomandibular disorders: review, criteria examinations and specifications, critique. J Craniomandib Disord 1992; 6: 301-355.

3. Cahlin BJ, Dahlström L. No effect of glucosamine sulfate on osteoarthritis in the temporomandibular joints - a randomized, controlled, short-term study. Oral Surg Oral Med Oral Pathol Oral Radiol Endod 2011; 112: 760-766.

4. Manfredini D, Rancitelli D, Ferronato G, Guarda-Nardini L. Arthrocentesis with or without additional drugs in temporomandibular joint inflammatory-degenerative disease: comparison of six treatment protocols. / Oral Rehabil 2012; 39: 245-251.

5. Guarda-Nardini L, Cadorin C, Frizziero A, Ferronato G, Manfredini D. Comparison of 2 hyaluronic acid drugs for the treatment of temporomandibular joint osteoarthritis. J Oral Maxillofac Surg 2012; 70: 2522-2530.

6. Palla S, Farella M. External validity: a forgotten issue? J Orofac Pain. 2009; 23: 297-298.

7. Manfredini D, Guarda-Nardini L, Winocur E, Piccotti F, Ahlberg J, Lobbezoo F. Research diagnostic criteria for temporomandibular disorders: a systematic review of axis I epidemiologic findings. Oral Surg Oral Med Oral Pathol Oral Radiol Endod 2011; 112: $453-462$.

Evidence-Based Dentistry (2013) 14, 5-7. doi:10.1038/sj.ebd.6400909 